\title{
Decompression via microsurgical anterior foraminotomy for cervical spondylotic myelopathy
}

\section{Technical note}

\section{Hae-Dong Jho, M.D., Ph.D.}

Department of Neurological Surgery, University of Pittsburgh School of Medicine, Pittsburgh, Pennsylvania

Over the past few years, a microsurgical anterior foraminotomy technique has been developed by the author and used to achieve spinal cord decompression for the treatment of cervical spondylotic myelopathy. A 5 X 8-mm unilateral anterior foraminotomy is accomplished by resecting the uncovertebral joint via an anterior approach. Through the foraminotomy hole, the posterior osteophytes at the spinal cord canal are removed diagonally up to the beginning of the contralateral nerve root. To treat multilevel disease, a tunnel is made among the foraminotomy holes. This technique accomplishes widening of the spinal cord canal in the transverse and longitudinal axes by direct resection of the compressive lesions through the holes of unilateral anterior foraminotomies; however, it does not require bone fusion or postoperative immobilization. Postoperatively patients remain in the hospital overnight, and do not need to wear cervical braces. This new surgical technique has shown excellent clinical outcomes with fast recovery and adequate anatomical decompression in patients with cervical spondylotic myelopathy. The surgical technique is reported and illustrated by two of the author's cases.

Key Words * cervical vertebrae * discectomy * intervertebral disc displacement * myelopathy * radiculitis * spine

Cervical spondylotic myelopathy has been surgically treated either by a posterior decompressive laminectomy or by an anterior discectomy or vertebrectomy approach.[1,2,5-9] Decompressive laminectomy is actually an indirect decompressive procedure because compressive lesions are most often located anteriorly.[2,6,9] An anterior vertebrectomy approach requires bone graft fusion and immobilization for a few months. After the author had accumulated experience with microsurgical anterior foraminotomy for cervical radiculopathy,[4] a new anterior technique was then developed to achieve direct decompression of the spinal cord without the use of bone fusion and immobilization.

This surgical technique is accomplished in a manner identical to the conventional anterior cervical approach. A unilateral microsurgical anterior foraminotomy is performed by removing the uncovertebral joint using a microsurgical drill. Through the foraminotomy hole, posterior osteophytes are removed diagonally providing decompression of the spinal cord up to the contralateral nerve root. Multiple foraminotomies are performed as needed. Posterior osteophytes, including the posterior portion of the 
vertebral bodies, are removed thereby creating a tunnel between foraminotomies. In doing so, the spinal canal is widened on the contralateral side and also through the longitudinal axis.

The clinical outcome has been satisfactory with fast recovery and minimal morbidity in more than 14 patients. This new surgical technique is reported with two illustrative cases.

\section{SURGICAL TECHNIQUE}

This surgical technique was studied and practiced on cadavers before it was applied in living patients. The goal was to achieve direct and effective anatomical decompression of the spinal cord with maintenance of spinal stability, thus eliminating the need for bone fusion and immobilization. The surgical technique of microsurgical anterior foraminotomy has been reported elsewhere.[4]

The operation is performed after general endotracheal anesthesia has been attained in the patient. Somatosensory evoked potentials (SSEPs) are obtained by stimulating the upper and lower extremities immediately after induction of general anesthesia. Once the baseline SSEPs are obtained, SSEP monitoring is used continuously until the end of the operation. Positioning of the patient is similar to that for the conventional anterior approach to the cervical spine. With the patient supine, a bolster is placed behind both shoulders to maintain gentle extension of the cervical spine. When the patient is properly positioned, the baseline spinal cord function is reconfirmed with SSEP monitoring. The head is positioned with the midline upright. Both shoulders are gently pulled and fixed caudally with tape to facilitate a lateral view of the cervical spine on intraoperative roentgenogram. A cervical traction device is not used. The entire anterior neck is prepared with antiseptic solution and draped.

A 3- to 6-cm long transverse incision is made at the anterior neck along a skin crease that is similar to the incision made for an anterior approach to the cervical spine. The skin incision is made ipsilaterally to the radiculopathy or to the narrower side of the spinal canal. The first two-thirds of this incision is made medially to the sternocleidomastoid muscle and the remaining one-third is kept lateral to the medial border of the sternocleidomastoid muscle. The subcutaneous tissue and the platysma muscle are incised along the line of the skin incision. The loose connective tissue layer under the platysma muscle is cleanly undermined to provide space to operate. A combination of sharp and blunt dissection is used to access the anterior column of the cervical spine to keep the carotid artery and the sternocleidomastoid muscle lateral and the strap muscle, trachea, and esophagus medial. The prevertebral fascia is opened, and the anterior column of the cervical spine is exposed. The correct level is then confirmed with a radiographic lateral view of the cervical spine. Up to this point, the procedure is similar to that for an anterior approach to the cervical spine.

An anterior cervical discectomy retractor system is then applied; only smooth-tipped retractor blades are used. Retraction naturally exposes the ipsilateral longus colli muscle rather than the midline anterior disc surface. An operating microscope is used at this stage. The medial portion of the longus colli muscle is excised to expose the medial parts of the transverse processes of the upper and lower vertebrae. The vertebral artery (VA) is located anterior to the C-7 transverse process and beneath the longus colli. Therefore, when operating at the C6-7 level, care must be taken not to injure the VA while removing the medial portion of the longus colli. Because the VA occasionally enters the transverse foramen at another level, the longus colli is incised carefully under the operating microscope. For operations above the C6-7 level, the VA is not exposed purposefully at this point. 


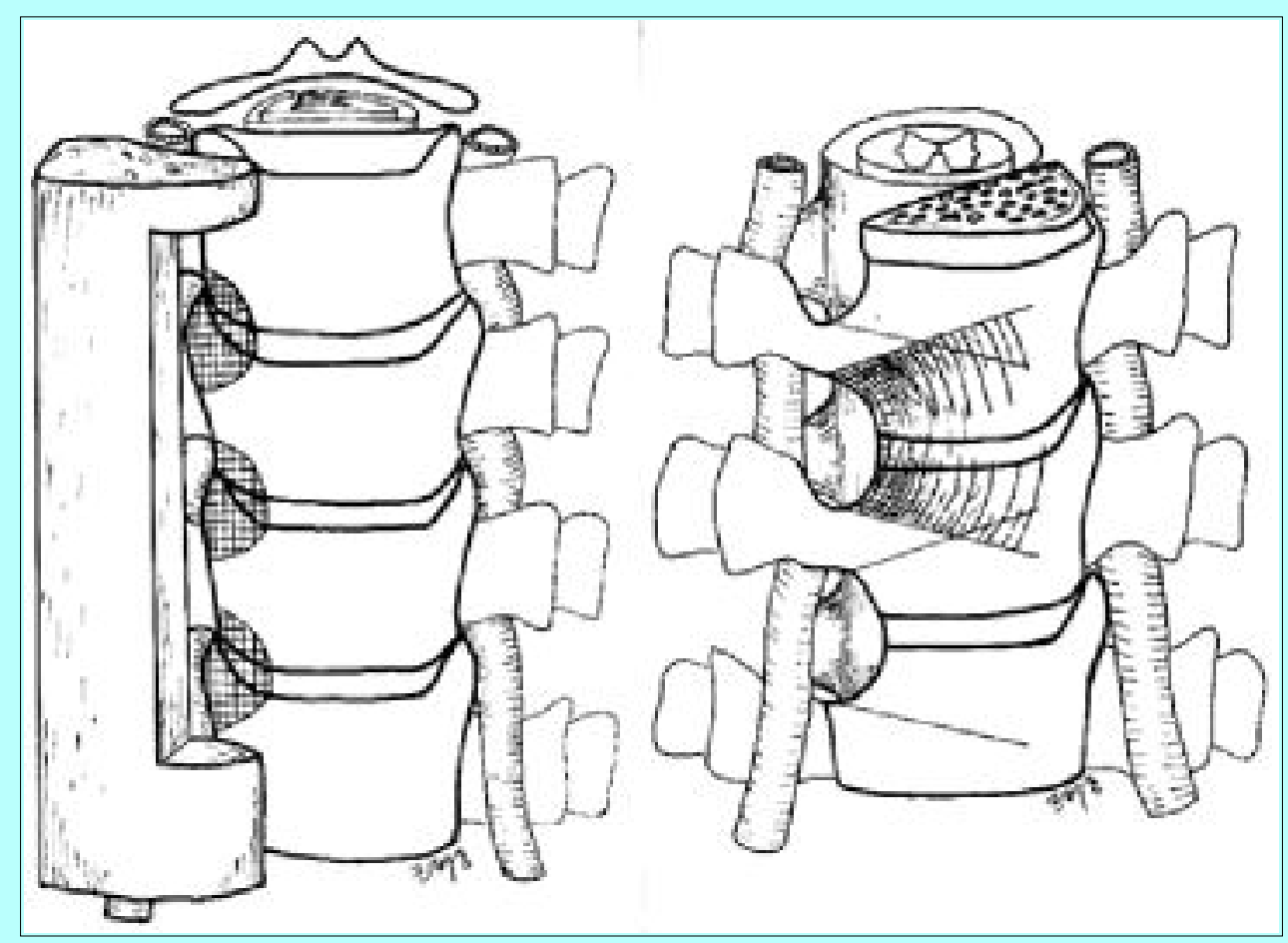

Fig. 1. Schematic drawings demonstrating the surgical approach. A transverse skin incision is made similar to that used in an anterior cervical discectomy. The unilateral longus colli muscle is exposed with anterior cervical discectomy retractors. The beginning of the transverse processes of corresponding levels of the cervical spine are exposed by resection of the medial portion of the longus colli muscle. The uncovertebral joint is located between the medial margins of the transverse processes (hatched areas [left]). The uncovertebral joint is removed with a high-speed microsurgical drill in the performance of the $6 \times 8 \mathrm{~mm}$ anterior foraminotomy. When the anterior foraminotomy is completed, decompression of the spinal cord canal is performed by resection of the posterior osteophytes across the midline diagonally to the beginning of the contralateral nerve root. The funnel-shaped hatched area indicates the direction of bone removal through the anterior foraminotomy hole (right). Longitudinal multilevel decompression is performed by making a tunnel through the foraminotomy holes.

Once the medial portions of the transverse processes of the upper and lower vertebrae have been identified, the ipsilateral uncovertebral joint between them can be seen; however, advanced spondylosis may obscure the anatomical landmark of the uncovertebral joint and transverse processes. Anterior spondylotic spurs at the intervertebral disc can act as a guide, leading to the uncovertebral joint superolaterally. Although the interface of the uncovertebral joint will be angled approximately $30^{\circ}$ cephalad from the horizontal line of the intervertebral disc in the normal cervical spine, advanced spondylotic changes may obscure the normal anatomy. The uncovertebral joint is drilled between the transverse processes using a high-speed microsurgical drill attached to an angled hand piece (Fig. 1). To prevent injury to the VA, a thin layer of cortical bone is left attached to the ligamentous tissue covering the medial portion of this artery. Drilling continues down to the posterior longitudinal ligament. As drilling advances posteriorly, the direction of the drill is gently inclined medially. When the posterior longitudinal ligament is exposed, a piece of thin cortical bone is left attached laterally to the periosteal and ligamentous tissue covering the VA. This lateral remnant of the uncinate process is dissected from the ligamentous tissue and fractured at the base of the uncinate process. It is further dissected from the surrounding soft tissue and removed, which enables identification of the VA by its pulsation between the 
transverse processes of the vertebrae. It is necessary to proceed cautiously with drilling at the base of the uncinate process because the nerve root lies just adjacent to it. After the uncinate process becomes loosened at its base, it is safer to remove the thin layer of remaining bone of the uncinate process by fracturing it rather than by continued drilling. When the remaining piece of the uncinate process is removed, the posterior osteophytes are drilled by crossing the midline diagonally toward the opposite margin of the spinal cord dura mater. The size of the hole made by the drilling at the uncovertebral joint is usually approximately 5 to $6 \mathrm{~mm}$ wide transversely and 7 to $8 \mathrm{~mm}$ vertically.

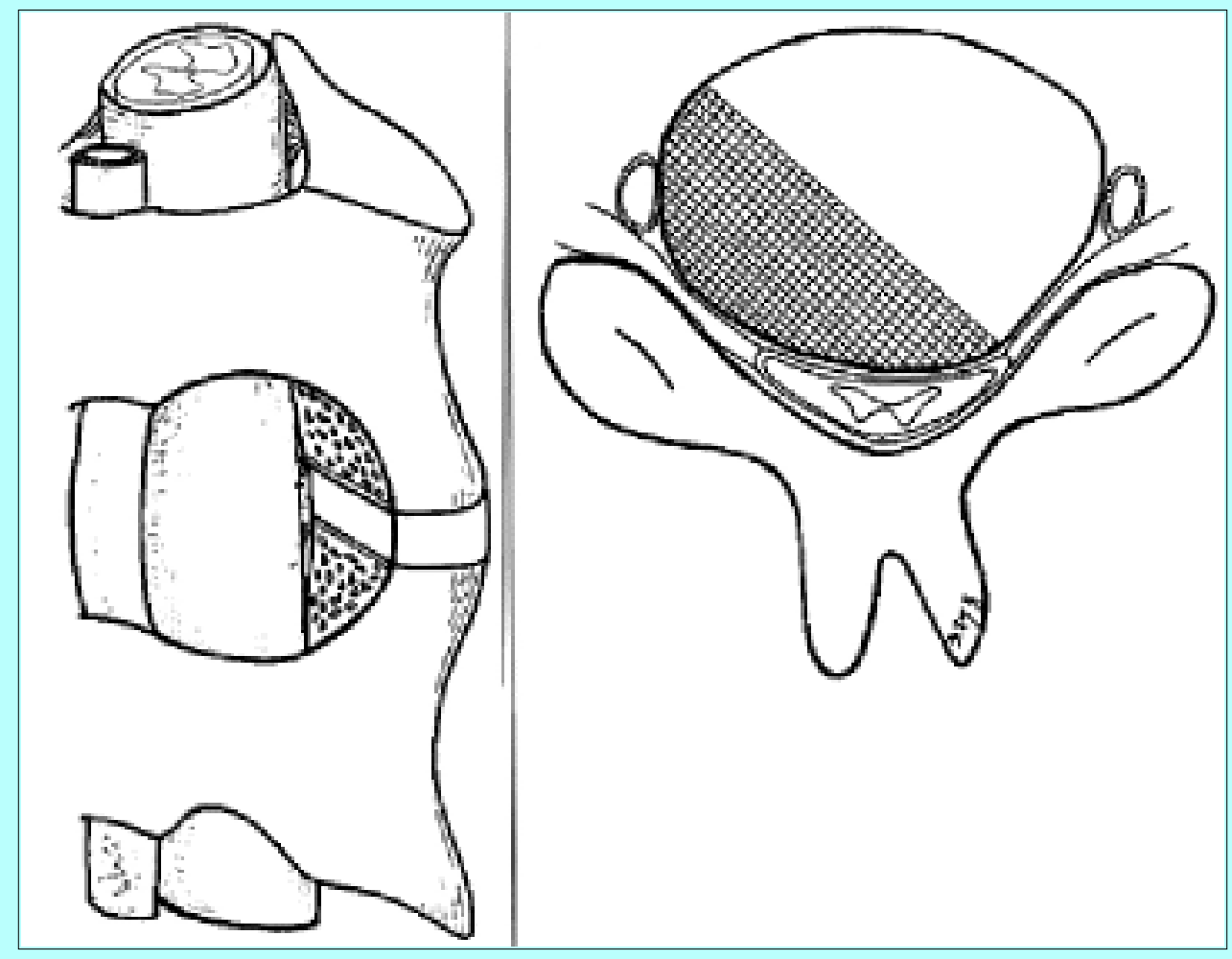

Fig. 2. Schematic drawings illustrating an oblique view through an anterior foraminotomy as seen with the operating microscope (left). Transverse decompression of the spinal cord canal is performed up to the beginning of the contralateral nerve root. A hatched area in the axial-view drawing is the area of the bone to be removed (right).

The posterior longitudinal ligament is incised and resected to achieve decompression of the ipsilateral nerve root and spinal cord. The beginning of the contralateral nerve root is identified for adequate decompression of the spinal canal in the transverse axis (Fig. 2). Multiple anterior foraminotomies are performed as needed. Using the holes of anterior foraminotomies, the spinal cord canal is enlarged in the longitudinal axis by removing the posterior portion of the vertebral bodies with Kerrison rongeurs and a long-armed up-biting curet. The bone bleeding is controlled with the application of bone wax. Epidural bleeding from the posterior longitudinal ligament can be controlled with bipolar coagulation. Hemostatic agents are not used in the epidural space.

Finally, the platysma is closed with interrupted No. 3-0 absorbable stitches, and the skin is approximated with subcuticular sutures. To minimize postoperative incisional pain, a local anesthetic (a few milliliters) is injected subcutaneously. A cervical collar is not used. Although microsurgical anterior foraminotomy for cervical radiculopathy has been performed as outpatient surgery, this group of patients with myelopathy stayed in the hospital overnight to observe their spinal cord function clinically; they were discharged home the next morning. 


\section{ILLUSTRATIVE CASES}

\section{Case 1}

This 54-year-old man was referred to the University of Pittsburgh Medical Center in June, 1995 with a 3 -year history of progressive radiculomyelopathy. Since sustaining a trivial injury to his neck 7 years earlier, he had suffered significant pain in his neck and both shoulders. For the past 3 years, he had experienced pain and paresthesia along both upper extremities, with the right being worse than the left, in addition to worsening of the pain in his neck and both shoulders. He also noticed a tingling sensation, tightness, and an aching pain in his right leg, a balance disorder causing frequent falling, and urinary urgency and frequency. He experienced an electric shocklike sensation along the right hemibody when he raised his hands over his head.

Examination. On examination, he had 4-/5weakness of his right deltoid and biceps muscles, $3+/ 5$ weakness in his left deltoid and biceps muscles, $4 / 5$ strength in the rest of the muscle groups of his right upper extremity, and 4-/5 strength in the remainder of the muscle groups of his left upper extremity. Touch and pinprick sensation and position and vibration sense in both upper extremities were preserved but decreased. His biceps reflexes were absent bilaterally. Triceps reflexes were hyperreflexic bilaterally. Hoffmann's signs were present bilaterally. Motor strength in both lower extremities were normal. Position and vibration sense in both lower extremities were present but diminished. Knee and ankle reflexes were hyperreflexic. Babinski signs were present bilaterally. He walked with a wide-based gait. His Romberg's test was positive.

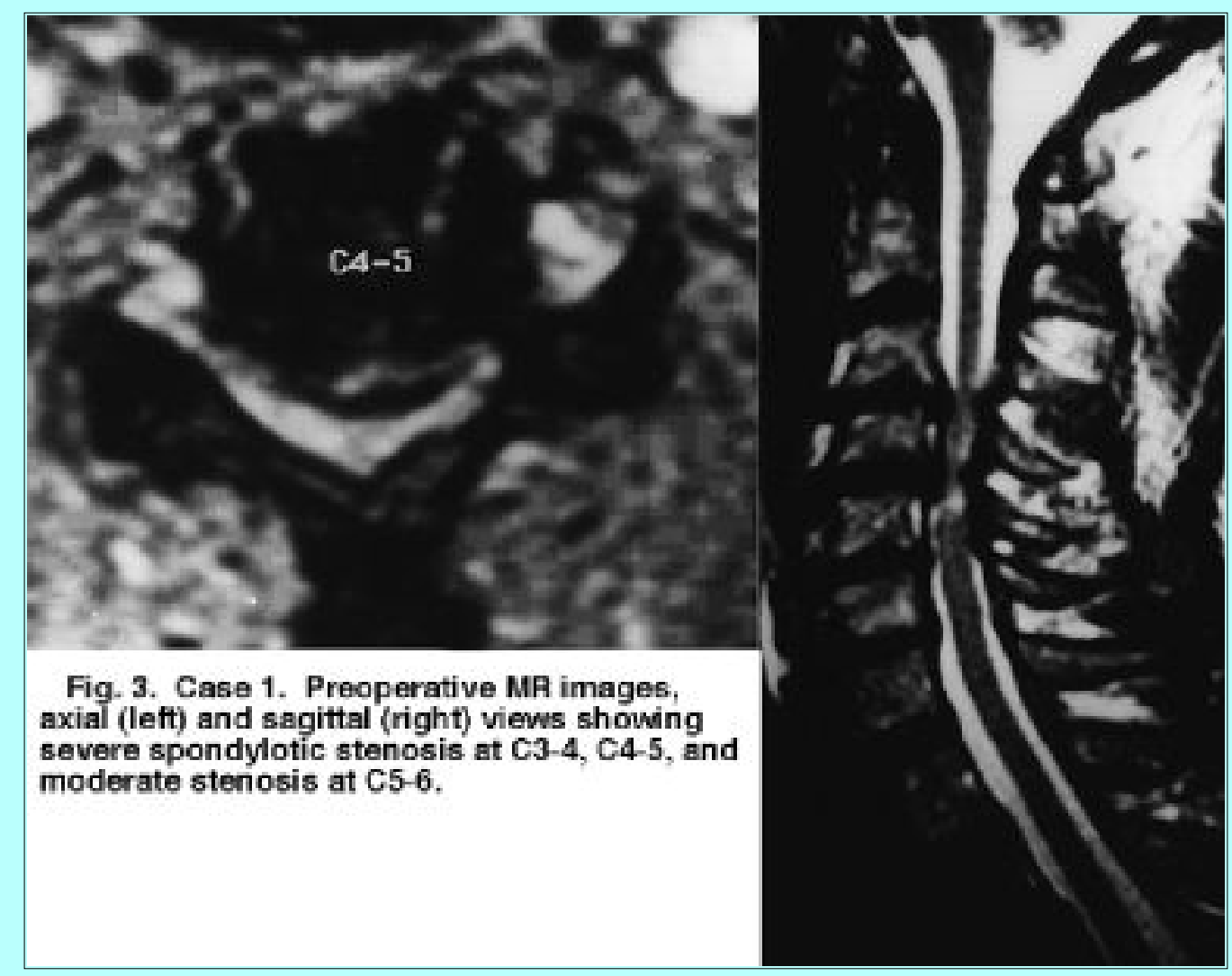

Magnetic resonance (MR) imaging of the cervical spine showed severe stenosis at C3-4, C4-5, and moderate stenosis at C5-6 with severe spinal cord compression and narrowing of the neural foramina (Fig. 3). 


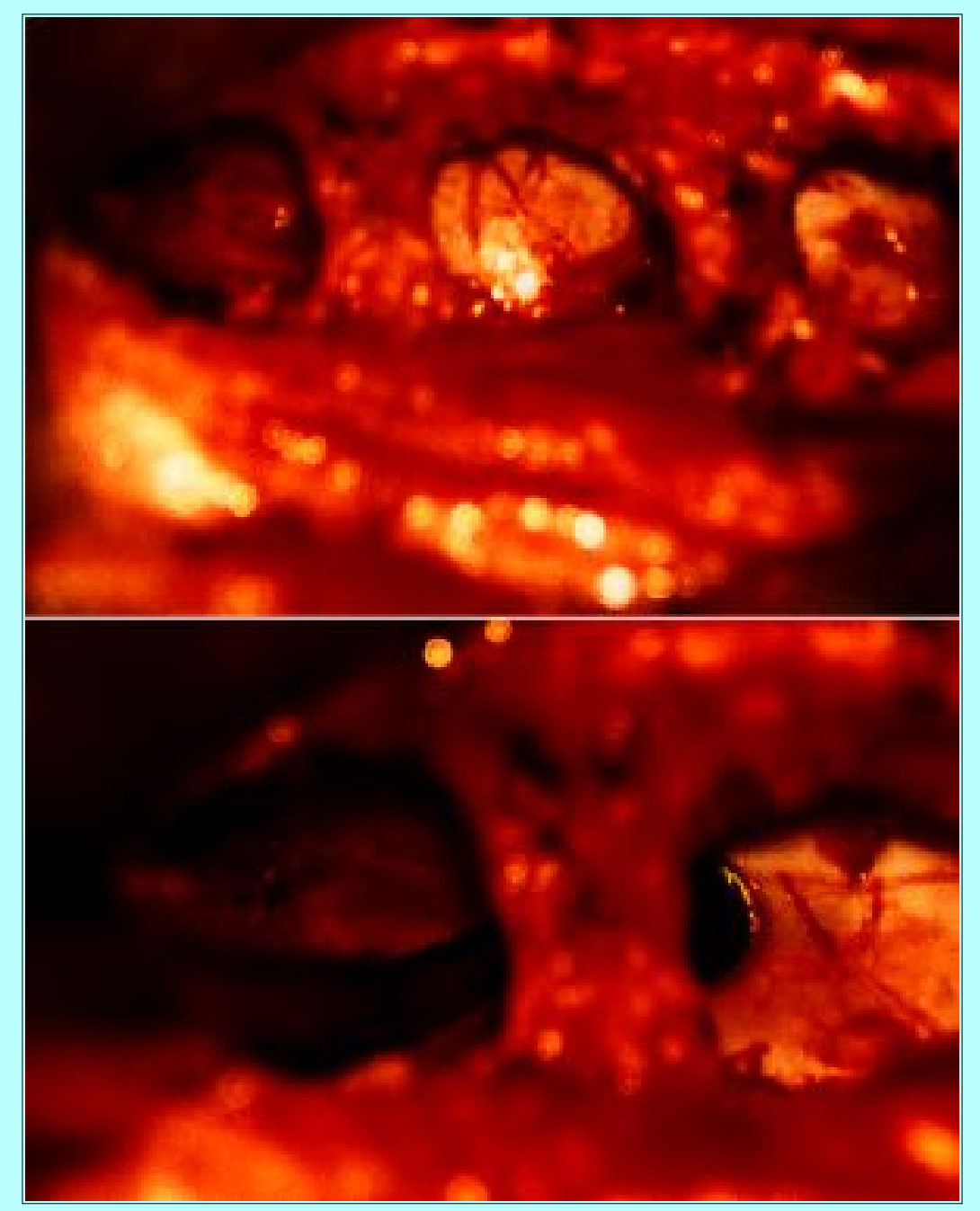

Fig. 4. Case 1. Intraoperative photographs showing the anterior view of the dura mater of the spinal cord through the anterior foraminotomy holes at C3-4, C4-5, and C5-6 (upper). An angled probe is passed behind the C-5 vertebral body after resection of the posterior spurs (lower).

Operation and Postoperative Course. The patient underwent spinal canal decompression via right-sided microsurgical anterior foraminotomy holes at C3-4, C4-5, and C5-6 (Fig. 4) and stayed overnight in the hospital postoperatively. He noticed improvement of his radicular symptoms and his balance disorder immediately after his operation. At a 6-week follow-up visit, his radiculomyelopathy had improved, leaving only residual hyperreflexia and stiffness of the neck. He continued to do well, with residual hyperreflexia, at his 1-year follow-up examination. Postoperative computerized tomography (CT) reconstructions and MR imaging of the cervical spine confirmed generous decompression of the spinal canal (Figs. 5-7). 


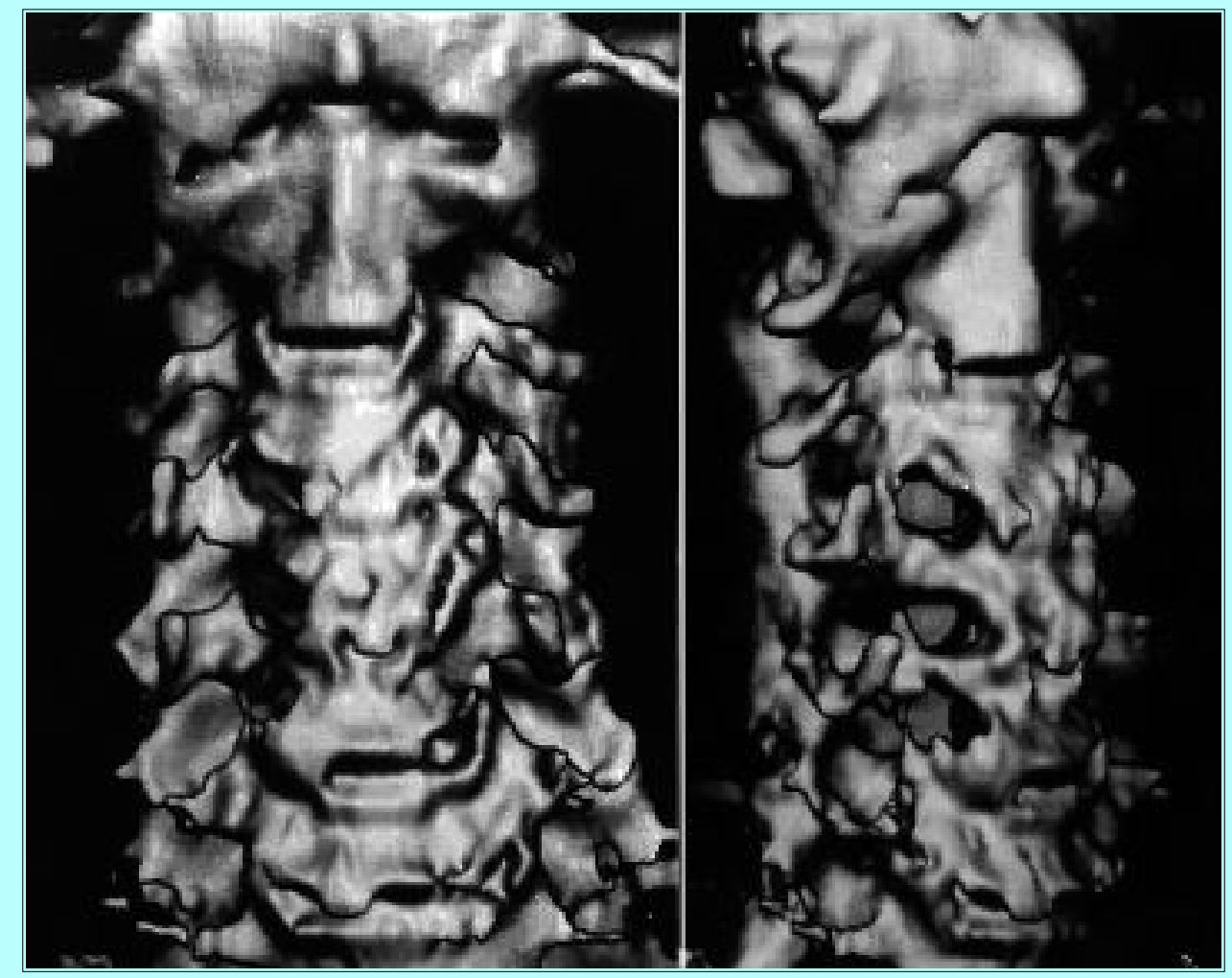

Fig. 5. Case 1. Postoperative CT reconstructions in the anteroposterior (left) and right oblique (right) views demonstrating right-sided anterior foraminotomy holes at C3-4, C4-5, and C5-6.

A flexion-extension dynamic roentgenogram of the cervical spine obtained on postoperative Day 1 and at 6 weeks postoperatively revealed a stable cervical spine.

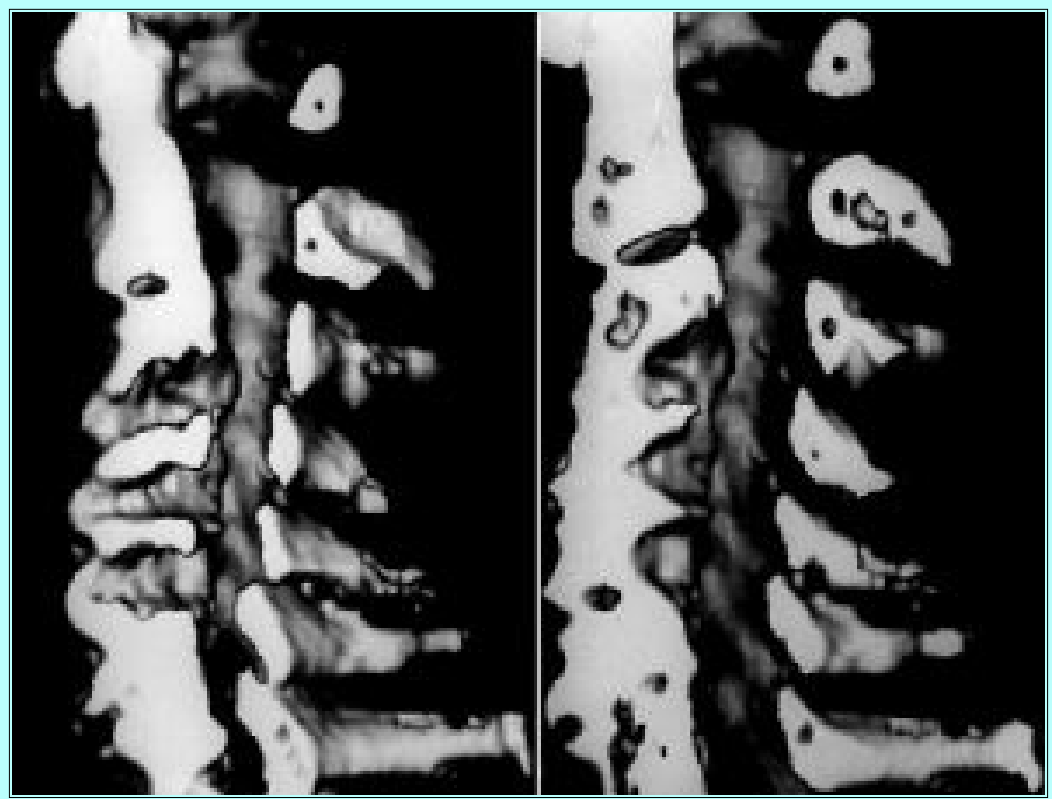

Fig. 6. Case 1. Postoperative CT reconstructions in the right paramedian (left) and median sagittal (right) views showing three-level anterior foraminotomy tracts and the dimension of spinal canal decompression.

\section{Case 2}

This 55-year-old man came to the University of Pittsburgh Medical Center with an 8-year history of 
progressive cervical myelopathy. Since developing severe neck pain after a dental procedure that he had undergone in 1987, he had experienced progressive pain and weakness in both of his upper extremities, the left side worse than right. He also experienced numbness and an electric shocklike sensation below his neck. For the past 6 months he had noticed progressive difficulty in maintaining his balance. He had been given two varying surgical opinions by two different spine surgeons, which included an anterior vertebrectomy, fusion, and instrumentation, or a posterior laminectomy followed by fusion and instrumentation. When he became concerned about the extensiveness of the operations, he decided to seek a third opinion.
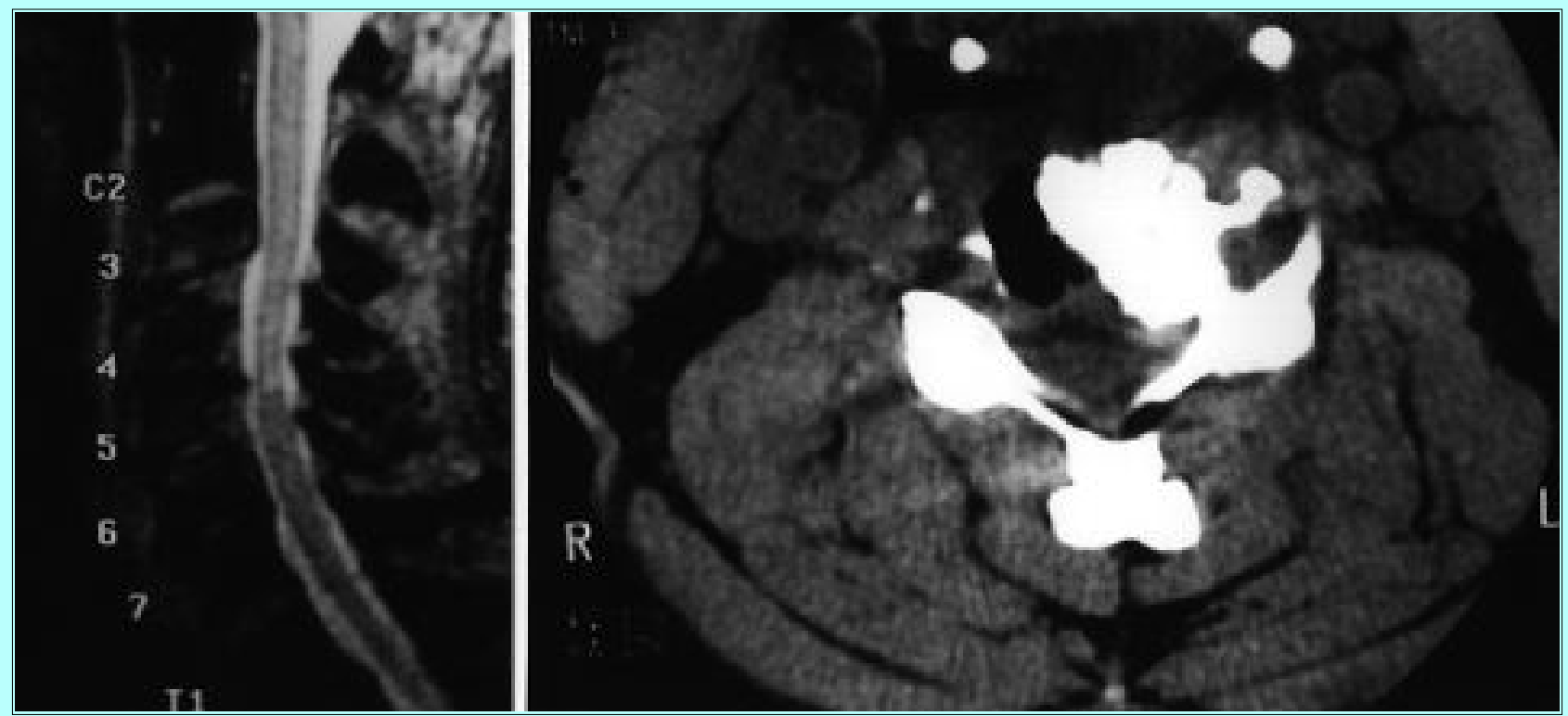

Fig. 7. Case 1. Postoperative sagittal MR (left) and axial CT images (right) demonstrating generous decompression of the spinal cord. Increased signal intensity in the central spinal cord at the C4-5 level can be noted in the T2-weighted sagittal MR image, which was present preoperatively.

Examination. On examination, he had restricted range of motion in his cervical spine at $50 \%$ less than normal. Motor strength in each individual muscle group was well maintained. Touch and pinprick sensation were diminished below C-4. Position and vibration sense in his upper and lower extremities were also diminished. His reflexes in both upper and lower extremities were all 4/4 and hyperreflexic. Hoffmann's signs were present bilaterally. Babinski signs were present bilaterally. Three beats of ankle clonus were present bilaterally. He walked with a wide-based gait. 


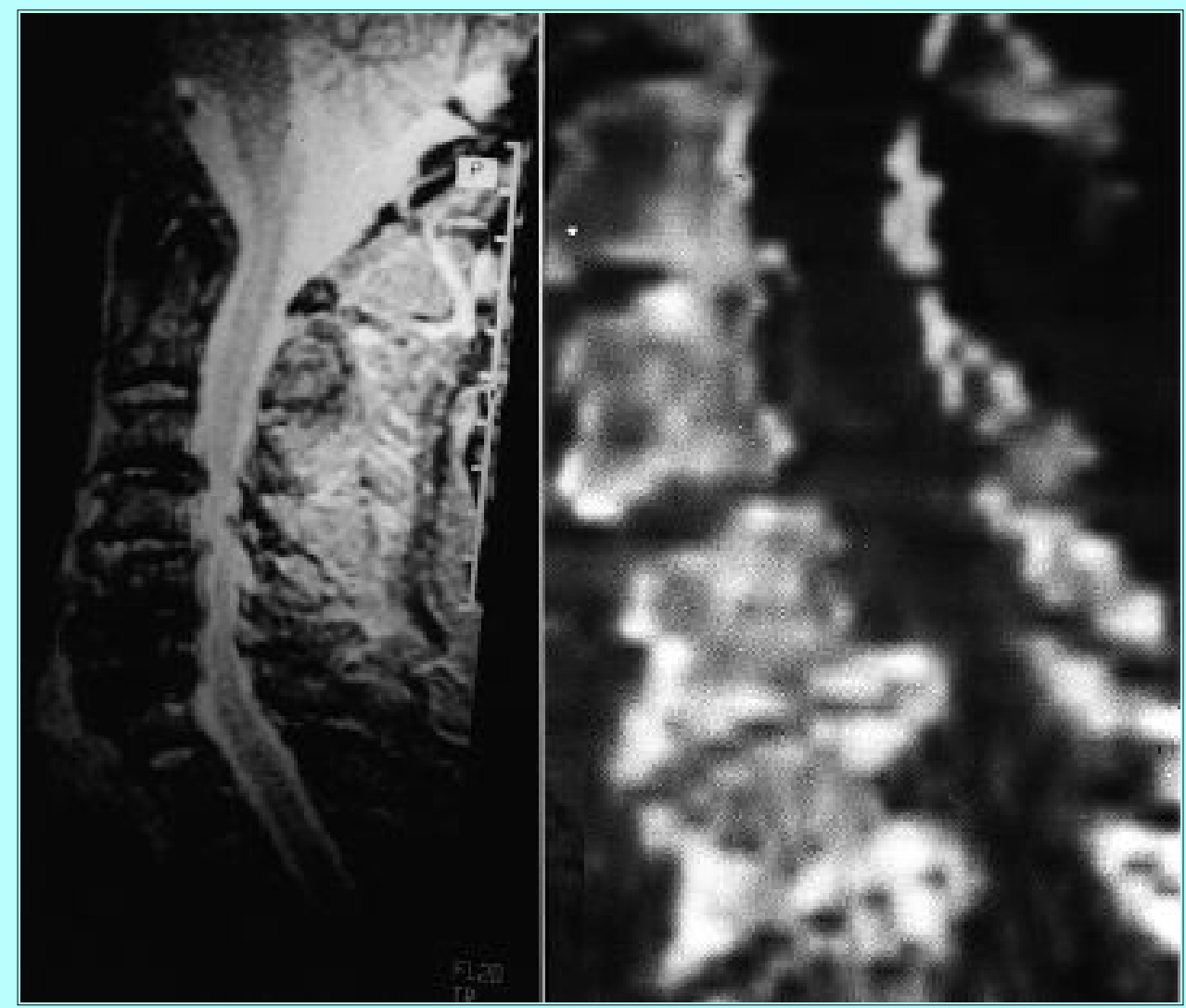

Fig. 8. Case 2. Preoperative MR image (left) and sagittal CT scan reconstruction (right) revealing C3-4, C5-6, and C6-7 spondylotic stenosis.

Magnetic resonance and CT images of the cervical spine showed spondylotic spurs at C3-4, C5-6, and C6-7 compressing the spinal cord (Fig. 8). The patient underwent spinal cord decompression via the right foraminotomy holes at C3-4, C5-6, and C6-7 in February 1995. He stayed overnight in the hospital postoperatively. His balance began to improve the day after his operation. During his 6-week follow-up visit, he was able to execute a tandem gait with complete resolution of his numbness and the electric shocklike sensation. Postoperative MR images obtained at 6 weeks demonstrated generous anatomical decompression (Fig. 9). Flexion-extension lateral views of a cervical spine roentgenogram taken on postoperative Day 1 and at 6 months confirmed stability without fusion. He continued to display hyperreflexia in his upper and lower extremities at the 6-week and 1-year follow-up examinations.

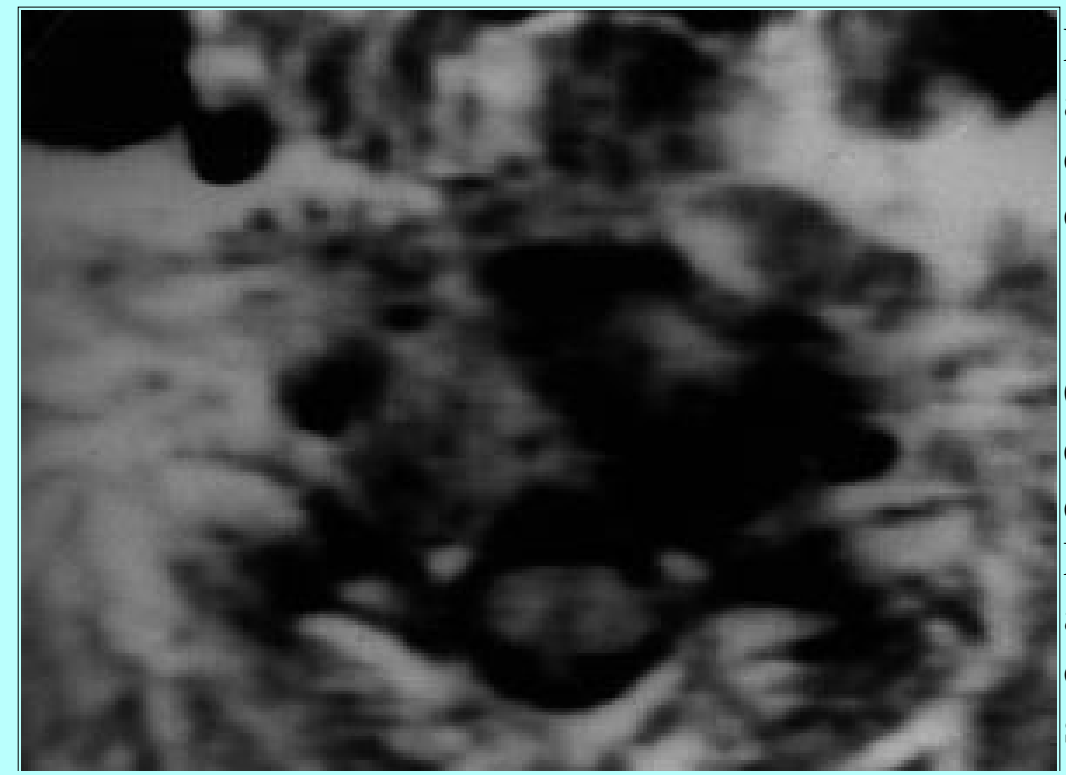

Fig. 9. Case 2. Postoperative MR images, axial (upper) and sagittal (lower) views demonstrating adequate anatomical decompression of the spinal cord.

\section{DISCUSSION}

Cervical spondylotic myelopathy has been treated either by posterior laminectomy or by anterior discectomy or vertebrectomy approaches.[1,2,5-9] Most surgeons use the posterior laminectomy approach without resection of the spondylotic spurs only to accomplish indirect decompression of the spinal cord because compressive lesions are most 


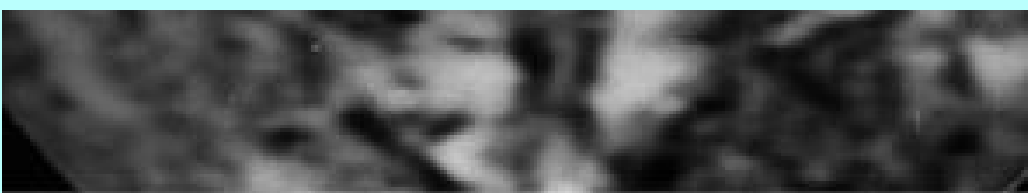

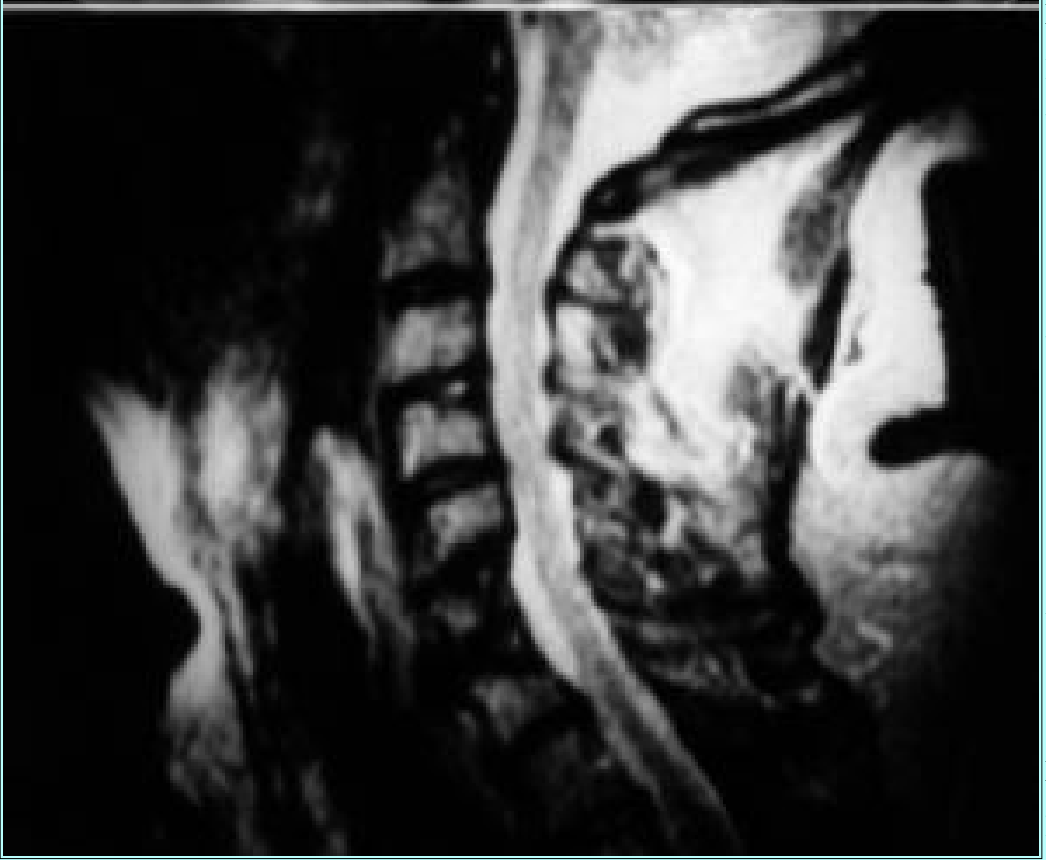

often located anteriorly.[9] The anterior approach incorporating bone fusion techniques was developed in the 1950s and has been technically refined with the development of spinal instruments. $[1,5,7,8]$ The anterior vertebrectomy technique requires bone fusion with or without spinal instrumentation. The technique reported here accomplishes direct decompression of the spinal cord canal as does the anterior vertebrectomy technique; however, it does not require bone fusion or postoperative immobilization. This technique is applicable only if the compressive lesion is located anterior to the spinal cord.

Anterolateral techniques have been reported with or without bone fusion. [3,10] Verbiest[10] used an anterolateral approach to excise lateral spurs by resection of the transverse processes and mobilization of the VA. He used this anterolateral technique for decompression of the VA, foraminal decompression, lateral disc herniation, and reconstruction of the postganglionic upper brachial plexus. He did not use this anterolateral approach for spinal cord decompression.

Hakuba[3] reported a transuncodiscal approach that was a combined anterior and lateral approach to a cervical disc. He removed posterior and lateral osteophytes in addition to the entire intervertebral disc with or without bone graft fusion. The author's technique differs from Hakuba's procedure in that the technique reported here uses only the unilateral anterior foraminotomy holes to resect compressive lesions and does not remove most of the intervertebral disc, which maintains spinal stability.

The author has used this technique in more than 14 patients to treat cervical spondylotic myelopathy. The operation time using this technique was comparable to that of microscopic anterior cervical discectomy. All 14 patients stayed overnight in the hospital, and none developed any complications. Postoperatively, all showed excellent improvement of their myelopathy. A flexion-extension roentgenogram of the cervical spine showed good stability in all patients on postoperative Day 1 as well as 6 weeks later. Although long-term follow-up data on the maintenance of the motion segments have not yet been gathered in this group of patients, the intervertebral motion segments are expected to be fused eventually by advanced spondylotic changes and by the surgical effect of substantial bone resection. Although most of the anterior intervertebral segments are preserved, resection of the posterior spondylotic spurs and vertebral bodies with this technique is still quite extensive. In addition, most of these patients had already experienced advanced spondylotic changes with limited motion at the involved intervertebral motion segments. The main advantages of this technique seem to be the patients' quick recovery and short hospital stay because of the elimination of bone fusion and immobilization. Although postoperative flexion-extension lateral roentgenograms of the cervical spine did not show spinal instability, the true biomechanical effect of this surgical procedure remains to be tested. Because this microsurgical procedure is technically demanding in the execution of adequate decompression of the spinal cord, it must be thoroughly practiced on cadavers before it is applied in patient treatment. 


\section{Acknowledgments}

The author wishes to thank Arthur P. Nestler, B.S.N., and Robin Coret, B.A., for their assistance with preparation of the manuscript.

\section{References}

1. Cloward RB: The anterior approach for removal of ruptured cervical disk. J Neurosurg 15:602-615, 1958

2. Fager CA: Results of adequate posterior decompression in the relief of spondylotic cervical myelopathy. J Neurosurg 38:684-692, 1973

3. Hakuba A: Trans-unco-discal approach. A combined anterior and lateral approach to cervical discs. J Neurosurg 45:284-291, 1976

4. Jho HD: Microsurgical anterior cervical foraminotomy for radiculopathy: a new approach to cervical disc herniation. J Neurosurg 84:155-160, 1996

5. Lunsford LD, Bissonette DJ, Zorub DS: Anterior surgery for cervical disc disease. Part 2: Treatment of cervical spondylotic myelopathy in 32 cases. J Neurosurg 53:12-19, 1980

6. Mixter WJ, Barr JS: Rupture of the intervertebral disc with involvement of the spinal canal. N Engl J Med 211:210-215, 1934

7. Robinson RA, Smith GW: Anterolateral cervical disc removal and interbody fusion for cervical disc syndrome. Bull Johns Hopkins Hosp 96:223-224, 1955 (Abstract)

8. Saunders RL, Bernini PM, Shirreffs TG, et al: Central corpectomy for cervical spondylotic myelopathy: a consecutive series with long-term follow-up evaluation. J Neurosurg 74:163-170, 1991 9. Scoville WB: Cervical spondylosis treated by bilateral facetectomy and laminectomy. J Neurosurg 18:423-428, 1961

10. Verbiest H: A lateral approach to the cervical spine: technique and indications. J Neurosurg 28:191-203, 1968

Manuscript received September 30, 1996.

Accepted in final form November 14, 1996.

Address reprint requests to: Hae-Dong Jho, M.D., Ph.D., Department of Neurological Surgery, Presbyterian University Hospital, Suite B-400, 200 Lothrop Street, Pittsburgh, Pennsylvania 15213. 